JIOM Nepal, Volume 41, Number 2, August 2019, page 4-7

\title{
Donor Factors Affecting Short Term Graft Outcome in Live Donor Kidney Transplantation
}

\author{
${ }^{1}$ Rabin Nepali, 'Dibya S Shah, ${ }^{2}$ Prem R Gyawali, ${ }^{2}$ Uttam Sharma, ${ }^{2}$ Pawan R Chalise \\ 'Department of Nephrology, ${ }^{2}$ Department of Urology and Kidney Transplant Surgery, Maharajgunj Medical Campus, \\ Tribhuvan University Teaching Hospital, Kathmandu, Nepal
}

\section{Corresponding author:}

Rabin Nepali, MD, DM Nephrology

Department of Nephrology, Maharajgunj Medical Campus, Tribhuvan University Teaching Hospital, Kathmandu, Nepal Email: rabinnepali@gmail.com

Submitted: Oct 28, 2018

Accepted : Jul 22, 2019

\begin{abstract}
Introduction

Since the beginning of renal transplant, the objective has been to increase the patient and graft survival, both short term and long term. Data relating to donor factors affecting short term survival at 6 months in live donor kidney transplantation has been scarce. This single center study tries to examine the predictors of short term graft outcome at six months in recipients of live donor kidney transplants and explore which donor characteristics are the most useful in predicting the post-transplant graft function in Nepalese population.
\end{abstract}

\section{Methods}

All patients who underwent kidney transplantation between May 2015 to July 2016 were included in the study. The patients who expired during follow were excluded. The clinical and laboratory parameters of the donors were recorded. The recipients were followed up for six months post transplantation. The eGFR of the recipients and the occurrence of rejection were recorded at the end of six months post transplantation.

\section{Results}

A total of 82 donor-recipient pairs underwent living donor renal transplantation at our hospital during the study period. One recipient who expired during follow up was excluded. The mean age of donor was $45.20 \pm 11.226$ years of which 55 (67.9\%) were female and 26 (32.1\%) were male. The mean eGFR of the donor calculated from Cockcroft Gault equation was $81.98 \pm 18.11$. The eGFR of the recipient at the end of six months post transplantation calculated form the MDRD equation was $67.76 \pm 20.94$. A total of 7 patients (8.6\%) had rejection that were biopsy proven. Only donor eGFR was found to be significantly associated with eGFR of the recipient at six months post transplantation $(p=0.034)$. Body mass index of the donor was significantly associated with rejection in the recipient at six months post transplantation $(p=0.011)$.

\section{Conclusion}

Our study demonstrates that the donor eGFR and body mass index are independent and important factors affecting the short term graft outcome at six months post transplantation.

Keywords: Body mass index, donor eGFR, graft outcome, renal transplantation

\section{INTRODUCTION}

7 he first organ transplant to result in long-term success was a living donor kidney transplant performed at the Peter Bent Brigham Hospital (now Brigham and Women's Hospital) in Boston in 1954. ' A surgical team led by Nobel Laureate Joseph Murray transplanted a kidney removed from Ronald Herrick into his identical twin brother Richard. Since then, the objective has been to increase the patient and graft survival, both short term and long term. The overall outcome after live donor transplantation is good, with reported recipient and graft survival at 5 years in excess of $90 \%$ and $80 \%$, respectively.
However, there is considerable variability in individual outcomes. Live donors as a proportion of all donors are increasing and now provide $40 \%$ of kidneys for transplantation in Australia and in the context of Nepal it is $100 \% .^{2}$ Thus, the factors that predict outcomes of live donor kidney transplants are also becoming increasingly relevant.

The outcome of a graft can be divided into short term and Long term. The risk of short term graft loss can further be divided into early, high-risk period (within first six months) and later period of constant low risk. ${ }^{3,4}$ Factors which affect graft outcomes in renal allograft recipients can be categorized into donor, 
recipient and immunological factors.

There have been a number of studies reporting the donor factors affecting graft outcome in the first year of transplantation and beyond. ${ }^{5}$ Factors that have been reported to influence the outcome after live donor kidney transplantation include pretransplant glomerular filtration rate (GFR), 6,7 HLA match, donor age $^{6,8,9}$ and gender, and the relationship between donor and recipient. However, data relating to donor factors affecting short term survival in six months in live donor kidney transplantation has been scarce. This single center study tries to examine the predictors of short term graft outcome at six months in recipients of live donor kidney transplants and explore which donor characteristics are the most useful in predicting the post-transplant graft function in Nepalese population.

\section{METHODS}

All patients who underwent kidney transplantation between May 2015 and July 2016 were included in the study. The patients who expired during follow up were excluded.

The demographic variables of the donor were age, gender and body mass index. The clinical variables were systolic blood pressure of the donor and estimated glomerular filtration rate (eGFR) of the donor calculated from Cockcroft Gault (CG) equation. The laboratory variables were level of HLA mismatch, serum LDL-cholesterol of the donor, serum creatinine of the donor and serum creatinine of the recipient at six month post transplantation. The outcome variables were eGFR at the end of six month calculated from MDRD (The Modification of Diet in Renal Disease) equation and the rejection episodes during six month of follow up.

All the study variables in the donor were collected after thorough history, physical examination and investigation during the evaluation process for the renal transplantation.

All the recipients were followed up regularly, weekly in the first month post-transplant, biweekly for months 1-3 and monthly for months 3-6. At the end of six month, serum creatinine was measured, eGFR calculated from MDRD equation and renal biopsy proven rejection episodes were recorded with treatment given, if any.

Data from pro forma sheets were transferred to data sheets of computer software program, Statistical Package for the Social Sciences (SPSS) version 20. Mean and standard deviation was used for continuous variables and proportion were used for categorical variables. Univariate analysis was ised to assess the effect of all the donor variables on the outcome. Multiple linear regression analysis was used to measure the individual effect all the independent variables on the outcome. All tests of significance were two-tailed and $\mathrm{p}$ value less than 0.05 was considered to be significant.

\section{RESULTS}

Total of 82 patients underwent renal transplantation during the study period. One patient expired during follow up. Remaining 81 donor-recipient pairs were included in the study. The characteristics of donor population is shown in table 1. The characteristics recipient population is shown in table 2 . There were two sensitized recipients. Both had weakly positive T-cell tissue crossmatch positive and doubtful negative B cell tissue cross match. They underwent plasmapheresis, a total of three sessions with Intravenous immunoglobulin (IVIG), 100 mg/ $\mathrm{kg}$ after each session of plasmapheresis prior to the transplantation. After results of negative cross match were obtained, they underwent kidney transplantation. Second kidney transplantation was performed in two $(2.4 \%)$ recipients.

The eGFR of the recipient at the end of six month post transplantation calculated form the MDRD equation was $67.76 \pm 20.94$. A total of seven recipients $(8.6 \%)$ had acute rejection which were confirmed by renal biopsy. Among them, four had acute cellular rejection and remaining three had acute antibody mediated rejection. All patients were treated according to their renal biopsy findings. Despite treatment, one graft was lost in a patient with acute antibody mediated rejection.

With multivariate linear regression analysis, only

Table 1. Demographics of donor factors

\begin{tabular}{lc}
\hline \multicolumn{1}{c}{ Characteristics } & Value(s) \\
\hline Age (years) & $45.38 \pm 11.4$ \\
Age group (years) & \\
18-30 & $10(12.3 \%)$ \\
$31-40$ & $18(22 \%)$ \\
$41-50$ & $20(24.6 \%)$ \\
$51-60$ & $27(32.9 \%)$ \\
>60 & $6(7.4 \%)$ \\
Sex & \\
Male & $25(30.86 \%)$ \\
Female & $56(69.1 \%)$ \\
Serum LDL (mmol/L) & $2.4 \pm 0.91$ \\
HLA mismatch & \\
0/6 & $13(16 \%)$ \\
$1-3 / 6$ & $48(59.3 \%)$ \\
$4-6 / 6$ & $20(24.7 \%)$ \\
HLA DRB1 mismatch & \\
0 & $26(32.1 \%)$ \\
1 & $43(53.1 \%)$ \\
2 & $12(14.8 \%)$ \\
Systolic BP (mm of Hg) & $119.9 \pm 12.2$ \\
BMI (kg/m $\left.{ }^{2}\right)$ & $24.47 \pm 3.2$ \\
eGFR (ml/min/1.73m $\left.{ }^{2}\right)$ & $81.98 \pm 18.11$ \\
\hline
\end{tabular}


Table 2. Demographics of recipient factors

\begin{tabular}{lc}
\hline \multicolumn{1}{c}{ Characteristics } & Value(s) \\
\hline Age (years) & $32.7 \pm 9.7$ \\
Age group (years) & $11(13.6 \%)$ \\
$18-30$ & $22(27.2 \%)$ \\
$31-40$ & $32(39.5 \%)$ \\
$41-50$ & $11(13.6 \%)$ \\
$51-60$ & $5(6.2 \%)$ \\
$>60$ & \\
Sex & $62(76.54 \%)$ \\
Male & $19(23.45 \%)$ \\
Female & $2(2.4 \%)$ \\
HLA sensitized patients & $2(2.4 \%)$ \\
Second transplantation & $67.76 \pm 20.94$ \\
eGFR at six months & $7(8.6 \%)$ \\
(ml/min/1.73m $\left.{ }^{2}\right)$ & \\
Rejection &
\end{tabular}

donor eGFR calculated from CG equation was found to be significantly associated with eGFR of the recipient at six month post transplantation $(p=0.034)$ and body mass index of the donor was significantly associated with rejection in the recipient at six months post transplantation ( $p=0.011$ ) (Table 3 and 4 ).

\section{DISCUSSION}

This study was done to identify the donor factors that affected the short term graft outcome in live renal transplant at six month post transplantation. This study showed that only donor eGFR and body mass index are important and independent correlates of short term graft outcome. Other factors such as donor age, donor sex, systolic blood pressure, serum LDL cholesterol level, HLA mismatch level did not have a significant association with short term graft outcome.

In our study, the donor eGFR calculated from CG equation was $81.98 \pm 18.11 \mathrm{ml} / \mathrm{min}$. The donor eGFR calculated form the CG equation was found to be an independent factor affecting graft outcome in live renal transplantation at six month post transplantation. It was in accordance with findings of Carmel M. Hawley et al in which 206 live donorrecipient pairs were retrospectively studied. ${ }^{10}$ The donor eGFR calculated from CG equation was $109 \pm 23 \mathrm{ml} / \mathrm{min}$. It showed the eGFR calculated for CG equation was significantly associated with eGFR of the recipient at six month post transplantation. Naim Issa et al in a retrospective study of 264 live donors, also showed significant association of eGFR of the donor with graft outcome at 2 years post transplantation. ${ }^{11}$ This is an important finding because it is measurable variable before embarking on the transplant procedure. So, potentially we can choose the donor according to the their eGFR and improve the short term graft outcome. We chose the CG equation for the donor because it is one of the easy available and reliable equation for normal individuals. So, we can calculate the donor eGFR in the outpatient setting itself in the situation of multiple donors.

The mean donor BMI was $24.47 \pm 3.2 \mathrm{~kg} / \mathrm{m}^{2}$ in our study. We found that short-term allograft survival for recipients of kidneys from donors with elevated BMI did not differ from outcomes for recipients of kidneys from normal weight live donors. The similar rate of allograft survival suggests that recipients of kidneys from obese live donors have the potential to enjoy the same high level of renal function in the short term as live donor renal transplant recipients in general. Interestingly, recipients of kidneys from obese donors were associated with acute rejection at six month post-transplant. Peter P. Reese et al, who studied 5304 live donors also found no association of $\mathrm{BMI}$ and graft outcome at one-year post-transplant. ${ }^{12}$ However, he did not find any significant differences in recipient acute rejection. Saravanan et al concluded obesity was associated with an increased risk for acute rejection in first month post transplantation. ${ }^{13}$

Table 3. Multivariable linear regression analysis showing donor variables associated with eGFR $\left(\mathrm{mL} / \mathrm{min} / 1.73 \mathrm{~m}^{2}\right)$ at six months post-transplantation

\begin{tabular}{lccc}
\hline \multicolumn{1}{c}{ Donor variables } & p value & \multicolumn{2}{c}{$95.0 \%$ confidence interval } \\
\cline { 3 - 4 } Age & & Lower bound & Upper bound \\
Sex & 0.259 & -0.815 & 0.223 \\
Systolic Blood pressure & 0.200 & -17.648 & 3.763 \\
BMI & 0.659 & -0.294 & 0.462 \\
HLA mismatch & 0.711 & -1.133 & 1.652 \\
HLA DRB1 mismatch & 0.235 & -4.995 & 1.24 \\
Serum LDL & 0.434 & -10.757 & 4.674 \\
eGFR, CG & 0.172 & -1.819 & 9.964 \\
\hline
\end{tabular}


Table 4. Multivariable linear regression analysis showing donor variables associated with rejection at six months post-transplantation

\begin{tabular}{lccc}
\hline \multirow{2}{*}{ Donor variables } & \multirow{2}{*}{ p value } & \multicolumn{2}{c}{$95.0 \%$ confidence interval } \\
\cline { 3 - 4 } & & Lower bound & Upper bound \\
\hline Age & 0.993 & -0.008 & 0.008 \\
Sex & 0.754 & -0.137 & 0.189 \\
Systolic Blood pressure & 0.181 & -0.002 & 0.010 \\
BMI & 0.011 & -0.049 & -0.007 \\
HLA mismatch & 0.434 & -0.066 & 0.029 \\
HLA DRB1 mismatch & 0.723 & -0.097 & 0.139 \\
Serum LDL & 0.429 & -0.126 & 0.054 \\
eGFR, CG & 0.664 & -0.004 & 0.006 \\
\hline
\end{tabular}

However, the difference was not significant at one year post transplantation. A study from H.H. Wang et al in 62 live renal transplant, also found that the recipients of kidneys from donors with a higher BMI had lower long-term graft survival with higher rates of acute rejection. ${ }^{14}$ They suggested that the recipients with low body mass index might need time to compensate adequate blood flow for the relative large graft, thus carrying a higher chance of rejection and worse graft outcome. ${ }^{14}$

Regarding other variables such as age, gender, LDL cholesterol, systolic blood pressure, HLA mismatch level, all have been found to have adverse effect on long term survival of the graft. However, our study was focused on the short term graft outcome of six months' post transplantation only. So, to see the effect of these variables in graft outcome longer follow up study might be necessary.

\section{CONCLUSION}

Our study demonstrates that the donor eGFR and body mass index are independent and important factors affecting the short term graft outcome at six month post transplantation.

\section{CONFLICT OF INTEREST}

None declared.

\section{REFERENCES}

1. Murray JE. Ronald Lee Herrick Memorial: June 15, 1931December 27, 2010. Am J Transplant 2011 and 11:419.

2. McDonald S EL, Chadban S. ANZDATA Registry 2004 Report. Available on-line at: http://www/. anzdata. org.au/anzdata/AnzdataReport/27thReport/files/ Ch08Transplantation.pdf.

3. Terasaki $P$, Cecka, JM, Gjertson, DW, et al. Risk rate and long-term kidney transplant survival. In: Clinical Transplants 1996, Terasaki, PI (Ed), UCLA Tissue Typing Laboratory, Los Angeles 1997, p. 443.
4. Prommool S JG, Cockfield SM, Halloran PF. Time dependency of factors affecting renal allograft survival. J Am Soc Nephrol 2000; 11:565.

5. Hariharan S, McBride MA, Cherikh WS, Tolleris $C B$, Bresnahan BA, Johnson CP. Post-transplant renal function in the first year predicts long-term kidney transplant survival. Kidney Int. 2002;62(1):311-318.

6. Norden G, Lennerling A, Nyberg G. Low absolute glomerular filtration rate in the living kidney donor: a risk factor for graft loss. Transplantation. 2000 and 70(9):1360-1362.

7. De La Vega LS TA, Bohorquez HE, et al. Patient and graft out- comes from older living kidney donors are similar to those from younger donors despite lower GFR. Kidney Int 2004 and 1654., 6.

8. Kwon OJ, Kwak JY, Kang CM. The impact of gender and age matching for long-term graft survival in living donor renal transplantation. Transplant Proc. 2005 and 37(2):726-728.

9. Velosa JA, Offord KP, Schroeder DR. Effect of age, sex, and glomerular filtration rate on renal function outcome of living kidney donors. Transplantation. 1995 and 60(12):1618-1621.

10. Hawley CM, Kearsley J, Campbell SB, et al. Estimated donor glomerular filtration rate is the most important donor characteristic predicting graft function in recipients of kidneys from live donors. Transplant International. 2007;20(1):64-72.

11. Issa $N$, Stephany $B$, Fatica $R$, et al. Donor Factors Influencing Graft Outcomes in Live Donor Kidney Transplantation. Transplantation. 2007;83(5):593-599.

12. Reese PP, Feldman HI, McBride MA, et al. Substantial variation in the acceptance of medically complex live kidney donors across US renal transplant centers. Am J Transplant 2008;8:2062-70.

13. Balamuthusamy $S$ PA, Zhang $R$, Florman $S$, Shenava

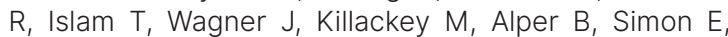
E, Slakey D, The Effects of Body Mass Index on Graft Survival in Adult Recipients Transplanted with Single Pediatric Kidneys. Am J Nephrol 2009;29:94-101.

14. Wang H-Hea. Size Does Matter-Donor-to-Recipient Body Mass Index Difference May Affect Renal Graft Outcome. Transplantation Proceedings.2012; Volume 44(Issue 1):267 - 269. 